\title{
Reduction of chemical networks
}

\section{The case of molecular clouds}

\author{
D. Wiebe ${ }^{1}$, D. Semenov ${ }^{2}$, and Th. Henning ${ }^{3}$ \\ 1 Institute of Astronomy of the RAS, Pyatnitskaya St. 48, 119017 Moscow, Russia \\ e-mail: dwiebe@inasan.rssi.ru \\ 2 Astrophysical Institute and University Observatory, Schillergäßchen 2-3, 07745 Jena, Germany \\ e-mail: dima@astro.uni-jena.de \\ ${ }^{3}$ Max Planck Institute for Astronomy, Königstuhl 17, 69117 Heidelberg, Germany \\ e-mail: henning@mpia.de
}

Received 20 September 2002 / Accepted 27 November 2002

\begin{abstract}
We present a new method to analyse and reduce chemical networks and apply this technique to the chemistry in molecular clouds. Using the technique, we investigated the possibility of reducing the number of chemical reactions and species in the UMIST 95 database simultaneously. In addition, we did the same reduction but with the "objective technique" in order to compare both methods. We found that it is possible to compute the abundance of carbon monoxide and fractional ionisation accurately with significantly reduced chemical networks in the case of pure gas-phase chemistry. For gas-grain chemistry involving surface reactions reduction is not worthwhile. Compared to the "objective technique" our reduction method is more effective but more time-consuming as well.
\end{abstract}

Key words. astrochemistry - stars: formation - molecular processes - ISM: molecules - ISM: abundances

\section{Introduction}

Despite the significant growth of computer power over the last years, the modelling of the chemical evolution of protostellar cores and protoplanetary discs is still a challenging computational task. This is even more true if one aims at the simultaneous self-consistent modelling of the dynamical and chemical evolution of these systems.

The mathematical formulation of the chemical processes in space is realised by differential equations describing chemical kinetics. They contain terms for all chemical reactions expected to proceed efficiently in the interstellar medium (ISM). Along with abundances of all involved species, these terms depend on the rate coefficients that are summarised in special databases. The relevant astrochemical rate files, like the UMIST rate file or Ohio New Standard Model, comprise hundreds of species and thousands of reactions (Millar et al. 1991, 1997; Le Teuff et al. 2000; Terzieva \& Herbst 1998; Aikawa \& Herbst 1999). Of course, one can only wonder if these databases contain all the information about reactions needed to accurately reproduce observed abundances of interstellar molecules. The opposite question to ask is if all this information is always needed.

In many astronomically interesting situations it is enough to follow the evolution of a limited number of species only.

Send offprint requests to: $\mathrm{D}$. Wiebe,

e-mail: dwiebe@inasan.rssi.ru
Intuitively, it seems to be apparent that to predict an abundance of $\mathrm{N}_{2} \mathrm{H}^{+}$, for example, one can afford computing $\mathrm{C}_{10}$ abundance with less accuracy or even ignore this molecule altogether. In most cases, though, modelling of the chemical evolution of a parcel of the interstellar gas under fixed conditions or in a limited predefined range of conditions is not very time-consuming, so one probably will not be eager to sacrifice any accuracy even for the sake of order of magnitude gain in computational speed. However, in the case of dynamical modelling with changing density, UV intensity, temperature etc., a reduced chemical network may help to distinguish between feasible and non-feasible problems or at least to ease the computational burden if parallel processors are in use. One possible application for reduced chemical networks would be the modelling of the evolution of magnetised protostellar clouds or protoplanetary accretion discs, when it is necessary to compute the fractional ionisation self-consistently. Another example of dynamically important species is carbon monoxide $(\mathrm{CO})$ which under certain conditions can be the most important molecular coolant (see, for example, Glassgold \& Langer 1973).

Several authors have already used reduced chemical networks, based on the above arguments, however, without presenting accurate mathematical considerations (e.g., Gerola \& Glassgold 1978; Howe et al. 1994; El-Nawawy et al. 1997). Recently, Ruffle et al. (2002) (hereafter RRPHH) and Rae et al. (2002) (hereafter RBHPR) made a first attempt to reduce 
astrochemical networks by so-called "objective reduction techniques", developed in combustion chemistry. They have shown that it is possible to quantify the reduction task by introducing a certain criterion that would allow in each particular case to indicate, abundances of which species must be followed in order to have a reasonable estimate for an abundance of a species under investigation. For static regions with physical conditions typical of diffuse clouds, they isolated the species set that contains tens instead of hundreds species. This reduction only introduces a factor of two uncertainty in predicted $\mathrm{CO}$ abundance. The same task has been solved for the fractional ionisation as well.

We perform a similar analysis of the UMIST 95 chemical reaction database in order to find if it is possible to reduce the number of species and/or reactions in the chemical networks used to calculate ionisation degree and carbon monoxide abundance under conditions typical of molecular clouds. Unlike RRPHH and RBHPR, we partly focus on the medium that is more advanced toward the formation of a star. This situation is more complicated from the chemical point of view. First, pre-stellar clouds are often shielded from the interstellar UV radiation field. Dissociating photons, being an additional chemical factor, in effect simplify the chemical network, as they prevent many complex species from being formed. Reactions on grain surfaces are another factor that cannot be ignored in dense clouds.

The paper is organised as follows. First, we describe our chemical model in Sect. 2. The species-based and reactionbased reduction methods are outlined in Sect. 3. Results of reductions obtained with both methods are presented in Sect. 4 (ionisation degree) and Sect. $5(\mathrm{CO})$. These results are discussed in Sect. 6. A conclusion follows.

\section{Chemical model}

We take the rates for gas-phase reactions from the UMIST 95 rate file (Millar et al. 1997). In addition to gas-phase chemistry, gas-dust interaction and dust surface reactions are considered in some of the models. The rates at which surface reactions proceed and the very applicability of the rate approximation to surface chemistry still remain a subject of debate. This is why many authors prefer to neglect surface reactions altogether (for instance, see Aikawa \& Herbst 1999; Bergin \& Langer 1997; Bergin et al. 1995; Charnley et al. 2001). However, in the attempt to constrain the chemical network in a cold and dense molecular cloud core, taking into account gas-dust interactions seems to be unavoidable, as dust is now widely believed to play a crucial role in molecular cloud chemistry, serving both as a sink for frozen-out species and as a catalyst for the synthesis of complex molecules (see, however, Turner 2000). In this paper we seek not to reproduce any particular abundance pattern observed in molecular clouds, but rather to investigate the very possibility of reduction when surface reactions are present. This is why we did not try to incorporate any new ideas about surface chemistry.
Table 1. Model designations.

\begin{tabular}{ll}
\hline \hline Notation & Meaning \\
\hline DIFF & $n_{\mathrm{H}}=10^{3} \mathrm{~cm}^{-3}$ \\
DENS & $n_{\mathrm{H}}=10^{7} \mathrm{~cm}^{-3}$ \\
GAS & Gas-phase network $\left(395 \times 3864^{\mathrm{a}}\right)$ \\
GAD & GAS with accretion and desorption \\
& $(543 \times 4593)$ \\
DUST & GAD with surface reactions $(543 \times 4785)$ \\
HM & "High metals" \\
LM & "Low metals" \\
\hline
\end{tabular}

aThe network contains 395 species involved in 3864 reactions.

Table 2. Adopted elemental abundances.

\begin{tabular}{lcc}
\hline \hline Element & "High metals" & "Low metals" \\
\hline $\mathrm{He}$ & $9.75(-2)$ & $9.75(-2)$ \\
$\mathrm{C}$ & $7.86(-5)$ & $7.86(-5)$ \\
$\mathrm{N}$ & $2.47(-5)$ & $2.47(-5)$ \\
$\mathrm{O}$ & $1.80(-4)$ & $1.80(-4)$ \\
$\mathrm{S}$ & $9.14(-6)$ & $9.14(-8)$ \\
$\mathrm{Si}$ & $9.74(-7)$ & $9.74(-9)$ \\
$\mathrm{Na}$ & $2.25(-7)$ & $2.25(-9)$ \\
$\mathrm{Mg}$ & $1.09(-6)$ & $1.09(-8)$ \\
$\mathrm{Fe}$ & $2.74(-7)$ & $2.74(-9)$ \\
$\mathrm{P}$ & $2.16(-8)$ & $2.16(-10)$ \\
$\mathrm{Cl}$ & $1.00(-7)$ & $1.00(-9)$ \\
\hline
\end{tabular}

\subsection{Physical conditions and initial abundances}

We solve equations of chemical kinetics for the range of fixed physical conditions, listed in Table 1, where model designations are given as well. Gas and dust temperatures are $10 \mathrm{~K}$ in all cases. No direct UV radiation is present, implying the conditions, typical of obscured regions, like cores of molecular clouds, where the visual extinction $A_{\mathrm{V}} \geq 10 \mathrm{mag}$. Dust grains are assumed to be silicate-like spheres with a constant size $a_{\mathrm{d}}=0.1 \mu \mathrm{m}$, density $\rho_{\mathrm{d}}=3 \mathrm{~g} \mathrm{~cm}^{-3}$, and $10^{6}$ surface sites for adsorption (Hasegawa et al. 1992). Dust is supposed to constitute $1 \%$ of the gas density by mass.

All abundances in the paper are given with respect to the number of hydrogen nuclei. We use "high metal" and "low metal" sets of initial abundances (e.g. Lee et al. 1998) quoted in Table 2. In the "high metal" set the standard solar elemental composition is corrected with a modest depletion of 2 for $S$ and stronger depletions of 10 for $\mathrm{Na}, 50$ for $\mathrm{Si}, 60$ for $\mathrm{Mg}$, and 110 for Fe. The "low metal" values contain additional depletion factors of 100 for each of these elements. The abundances of all elements but $\mathrm{P}$ and $\mathrm{Cl}$ are taken from Aikawa et al. (1996). For $\mathrm{P}$ and $\mathrm{Cl}$ we take values from Grevesse \& Sauval (1998) and use the same depletion factors as for Fe. Hydrogen is assumed to be completely in molecular form initially. All other elements are present in atomic form at $t=0$.

Below details of the adopted model of gas-dust interaction are provided. 


\subsection{Gas-grain interactions}

\subsubsection{Neutral accretion}

The accretion rate $k_{\mathrm{ac}}(i)$ of the $i$ th species is given by

$k_{\mathrm{ac}}(i)=\pi a_{\mathrm{d}}^{2} v_{\mathrm{th}}(i) n_{\mathrm{d}} S$,

where $v_{\text {th }}(i)=\sqrt{8 k T_{\text {gas }} / \pi m_{i}}$ is the thermal velocity for the $i$ th species, $n_{\mathrm{d}}$ is the number of dust grains per unit volume, $T_{\text {gas }}$ is the gas temperature, $m_{i}$ is the atomic mass of the $i$ th species, and $k$ is the Boltzmann constant. The sticking probability $S$ is assumed to be 0.3 for all neutral species except for $\mathrm{H}, \mathrm{He}$, and $\mathrm{H}_{2}$. The sticking coefficient of atomic hydrogen is estimated from Eq. (3.7) by Hollenbach \& McKee (1979). Sticking probabilities for helium and molecular hydrogen are assumed to be zero.

\subsubsection{Dissociative recombination on grain surfaces}

Following Umebayashi \& Nakano (1980), we suppose that all grains have unit negative charge and thus can act as electron donors in the same reactions of dissociative recombination that occur in the gas phase. The rate coefficient for reactions of dissociative recombination on grain surfaces is given by

$k_{\mathrm{dr}}(i)=\sum_{j} \alpha_{j} \pi a_{\mathrm{d}}^{2} v_{\mathrm{th}}(i) n_{\mathrm{d}} C_{\mathrm{ion}}$,

where

$C_{\text {ion }}=S\left(1+\frac{1.671 \times 10^{-3}}{a_{\mathrm{d}} T_{\mathrm{d}}}\right)$

with $a_{\mathrm{d}}$ and $T_{\mathrm{d}}$ being the dust size and temperature, respectively (Rawlings et al. 1992). Here the summation is over different recombination channels; the quantity $\alpha_{j}$ denotes the probability of a particular channel. Sticking probability $S$ is 0.3 for all ions. Products of these reactions are assumed to return to the gasphase immediately.

\subsubsection{Desorption from grain surfaces}

The two desorption processes taken into account in our model are thermal evaporation and cosmic ray induced desorption.

The thermal evaporation rate $k_{\mathrm{th}}(i)$ of the $i$ th surface species is

$k_{\mathrm{th}}(i)=v_{0} \exp \left(-T_{\mathrm{D}}(i) / T_{\mathrm{d}}\right)$

where $T_{\mathrm{d}}$ is the dust temperature, $k T_{\mathrm{D}}(i)$ is the binding energy for physical adsorption of the $i$ th species to the dust surface, $v_{0}$ is the characteristic vibrational frequency for the adsorbed species (Eq. (3) from Hasegawa et al. 1992).

The cosmic ray desorption rate $k_{\mathrm{cr}}(i)$ of the $i$ th surface species can be estimated as

$k_{\mathrm{cr}}(i)=3.16 \times 10^{-19} v_{0} \exp \left(-T_{\mathrm{D}}(i) / 70 \mathrm{~K}\right)$.

The values of $T_{\mathrm{D}}$ for most species are taken from Hasegawa \& Herbst (1993). There are some species in the UMIST 95 database for which desorption energies are not given in that paper. For these species, $T_{\mathrm{D}}$ is interpolated from values of chemically identical species or estimated as

$T_{\mathrm{D}}=50 A_{i}$

with $A_{i}$ being the corresponding atomic number, which is a good approximation for species from Table 4 of Hasegawa \& Herbst (1993).

\subsubsection{Surface reactions}

The surface reactions as well as their rates are taken from Hasegawa et al. (1992) with the appropriate correction for different densities. Activation energy of $1000 \mathrm{~K}$ is adopted for O + CO surface reaction (Hasegawa \& Herbst 1993).

\subsection{Chemical networks}

Three chemical networks are considered. The first one is the pure gas-phase network GAS. It consists of electrons, 12 atoms, 137 molecules, and 245 ions (395 species in total) involved in 3864 gas-phase reactions. The gas-grain network GAD is supplemented by 148 surface species and 729 gas-grain interaction processes (accretion, desorption, and surface ion recombination). The gas-grain network DUST contains 192 additional surface reactions. These networks form the basis of our investigations. In the rest of the text we refer to them as "full" networks. Equations of chemical kinetics are solved with the standard DVODE solver. Adopted solver parameters are given in the appendix.

\section{Reduction methods}

To reduce the number of species and/or reactions in a chemical network, one has to provide a mathematical method designed to estimate the significance of particular species or reactions to the evolution of the species under consideration. In this paper we elaborate two such methods that are outlined in this section.

\subsection{Species-based reduction}

This technique is derived from the method used by RRPHH and RBHPR. They have shown that it is possible to reduce the number of species in a chemical network in order to compute abundances of selected species with a reasonable accuracy. The species-based reduction technique is based on the strategy to specify important species and then to select from the entire set only necessary species, that must be included in the chemical network in order to compute abundances of important species with a reasonable accuracy.

Initially, the reduced species set consists of $N_{0}$ important species only. For each species in the entire set we compute the value

$B_{i}=\sum_{j=1, N_{0}}\left(\frac{n_{i}}{g_{j}} \frac{\partial f_{j}}{\partial n_{i}}\right)^{2}$ 


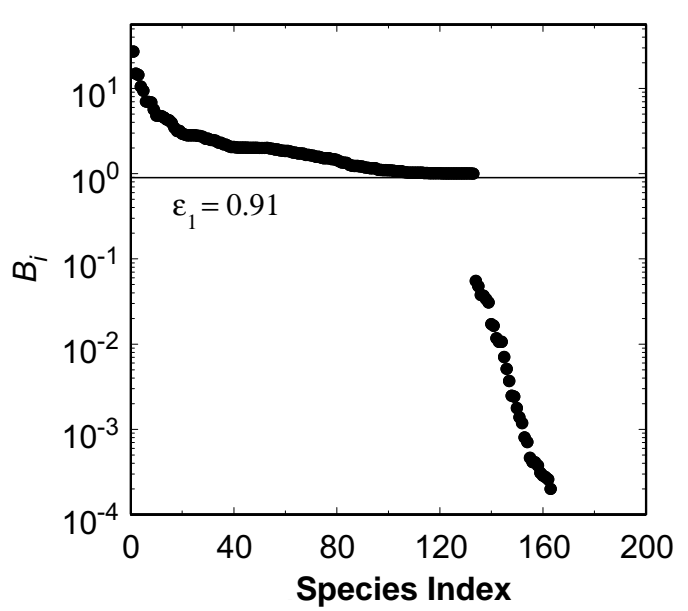

Fig. 1. Representative $B$ values for the HM-GAS-DIFF model computed at $t=10^{7}$ years. Important species are $\mathrm{Mg}^{+}$and $\mathrm{S}^{+}$. The value of the cut-off parameter $\epsilon_{1}$ is indicated.

where $n_{i}$ is the abundance of $i$ th species,

$f_{j}=\sum_{l m} k_{l m} n_{l} n_{m}-n_{j} \sum_{l} k_{j l} n_{l}=G_{j}-L_{j}$

is the net rate of the $j$ th species abundance change which can be expressed as the difference between net gain $G_{j}$ and loss $L_{j}$ rates, and

$g_{j}=\max \left(G_{j}, L_{j}\right)$

With these definitions, the quantity

$\frac{n_{i}}{g_{j}} \frac{\partial f_{j}}{\partial n_{i}}$

is the total rate of the abundance change of species $j$ due to those reactions that involve species $i$ only. The $B_{i}$ is a measure of the sensitivity of the selected species abundances to the abundance of the $i$ th species. As RRPHH noted, in this set of $B_{i}$ values there is a clear boundary between "needed" and "notso-needed" species. This is illustrated in Fig. 1, where representative $B_{i}$ values and a cut-off threshold $\epsilon_{1}$ are shown. We select only those species from the entire set that have $B_{i}$ above a boundary value, that is

$B_{i} \geq \epsilon_{1}$

They are added to the reduced set if they are not there already, and $B$ calculation is repeated with the expanded species set. The process goes on until at some step no species are added to the reduced set. The entire network then consists of these species and of only those reactions that involve the selected species. Normally, the resultant reduced set is a combination of several intermediate sets constructed at different times to account for "early" chemistry, "late" chemistry etc. But it turned out that the set reduced at the last time step $\left(t=10^{7}\right.$ years) works quite well at earlier times as well.

It should be kept in mind that this method represents only the first part of the algorithm proposed by RRPHH.

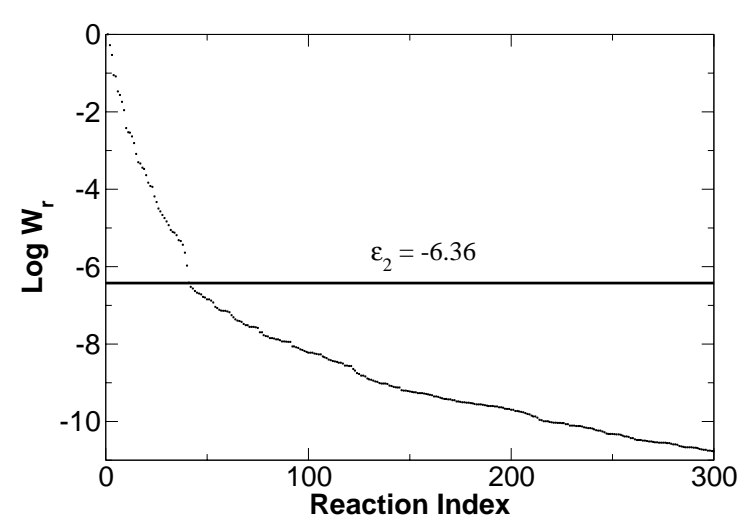

Fig. 2. Representative $w_{\mathrm{r}}$ values for the HM-GAS-DENS model computed at $t=10^{7}$ years. An important species is CO. The value of the cut-off parameter $\epsilon_{2}$ is indicated.

\subsection{Reaction-based reduction}

This new method allows to select only those species and reactions from the entire network that are necessary to compute abundances of important species with a reasonable accuracy. Unlike the species-based reduction technique, where the number of reactions is decreased only as a by-product of the lower number of species, in this method the numbers of unnecessary species and ineffective reactions are reduced simultaneously. In addition to the significantly shortened computational time, this could be useful if one aims at key destruction and formation pathways for important species, where a smaller number of chemical reactions makes the analysis easier.

The basic idea of the reaction-based reduction is to search for the production and destruction reactions, most important for the evolution of an important species, and to determine their relative significance. This is done via the analysis how sensitive the net formation (or loss) rate of a given species is to the presence of particular reactions at a certain physical time.

At the beginning, the chemical model is computed with the full network in order to obtain abundances of all species from the network during the entire evolutionary time span.

Second, the important species are specified. Further, the algorithm estimates the weights of all species $w_{\mathrm{s}}$ and reactions $w_{\mathrm{r}}$ in order to quantify their importance for the evolution of the species under investigation by the following iterative process.

At the first iteration, weights of important species are set to 1 and weights of all other species are set to 0 . At the $i$ th iteration all possible formation and destruction pathways of the current species $s_{i}$ are found and their significance values $w_{\mathrm{r}}^{i}$ are specified as

$w_{\mathrm{r}}^{i}(j)=\max \left\{w_{\mathrm{r}}^{i-1}(j), \frac{k_{j} n_{r_{1}}(j) n_{r_{2}}(j)}{\sum_{l=1, N_{\mathrm{r}}(i)} k_{l} n_{r_{1}}(l) n_{r_{2}}(l)} w_{\mathrm{s}}(i)\right\}$.

Here $k_{j}$ is the rate of the $j$ th reaction, $n_{r_{1}}(j)$ and $n_{r_{2}}(j)$ are the abundances of the first $r_{1}(j)$ and second $r_{2}(j)$ reactants in the $j$ th reaction, respectively, $N_{\mathrm{r}}(i)$ is the number of reactions, in which $s_{i}$ is a reactant or a product, and $w_{\mathrm{s}}(i)$ is the weight of the species $s_{i}$. 
Table 3. Reductions made for ionisation degree in a diffuse cloud.

\begin{tabular}{lllc}
\hline \hline Model & $\begin{array}{l}\text { Important } \\
\text { species }\end{array}$ & $\begin{array}{l}\text { Reduced } \\
\text { network }\end{array}$ & $\begin{array}{c}\text { Speed } \\
\text { gain }\end{array}$ \\
\hline \multicolumn{4}{c}{ Species-based reduction } \\
\hline HM-GAS-DIFF & $\mathrm{S}^{+}$ & $123 \times 1583$ & $\sim 25$ \\
HM-GAS-DIFF & $\mathrm{S}^{+}, \mathrm{Mg}^{+}$ & $126 \times 1619$ & $\sim 20$ \\
LM-GAS-DIFF & $\mathrm{H}_{3}^{+}$ & $282 \times 2539$ & $\sim 4$ \\
LM-GAS-DIFF & $\mathrm{H}_{3}^{+}, \mathrm{HCO}^{+}$ & $309 \times 3177$ & $\sim 3$ \\
\hline \multicolumn{4}{c}{ Reaction-based reduction } \\
\hline HM-GAS-DIFF & $\mathrm{e}^{-}$ & $58 \times 111$ & 550 \\
LM-GAS-DIFF & $\mathrm{e}^{-}$ & $73 \times 169$ & 490 \\
HM-DUST-DIFF & $\mathrm{e}^{-}$ & $210 \times 683$ & 15 \\
LM-DUST-DIFF & $\mathrm{e}^{-}$ & $357 \times 1648$ & 2 \\
\hline
\end{tabular}

Consequently, a new set of species, which are found at that iteration to be necessary for the evolution of the important species and which were not considered at previous iterations, is formed. Their weights $w_{\mathrm{s}}$ are estimated as the maximum possible values of the weights $w_{\mathrm{r}}$ of the reactions they are involved in. Then these species are added to the set of necessary species, and the last two steps are repeated. This process is performed for several stages during the computational time.

The iterations are stopped when all species and all time moments are considered. Then one obtains a reduced chemical network from the full network by choosing only those reactions, that have weights $w_{\mathrm{r}}$ exceeding a pre-defined cut-off threshold $\epsilon_{2}$

$\log w_{\mathrm{r}} \geq \epsilon_{2}$

The typical $w_{\mathrm{r}}$ values and cut-off parameter $\epsilon_{2}$ are shown in Fig. 2. The value of this cut-off is chosen to satisfy the requested accuracy requirement. In our calculations the maximum allowed error is set to $30 \%$. If there is a difference greater than $30 \%$ in abundances of the important species computed with the full and reduced networks for any particular time step, the cut-off is readjusted to a new, smaller value, and the last step is repeated.

\section{Ionisation degree}

We use the two reduction techniques described in the previous section to build the reduced chemical networks that could be used for the computation of the ionisation degree in molecular clouds. All the models are first computed with the full chemical networks. Then we apply the reduction techniques and compare the ionisation degree computed with the full and reduced networks. The results of the reduction together with relevant computational speed gains are summarised in Tables 3-5.

\subsection{Diffuse cloud}

This case corresponds to the cloud at the initial stage of the protostellar collapse $\left(n_{\mathrm{H}}=10^{3} \mathrm{~cm}^{-3}\right)$. For pure gas-phase chemistry and high initial metal abundances (HM-GAS-DIFF), the
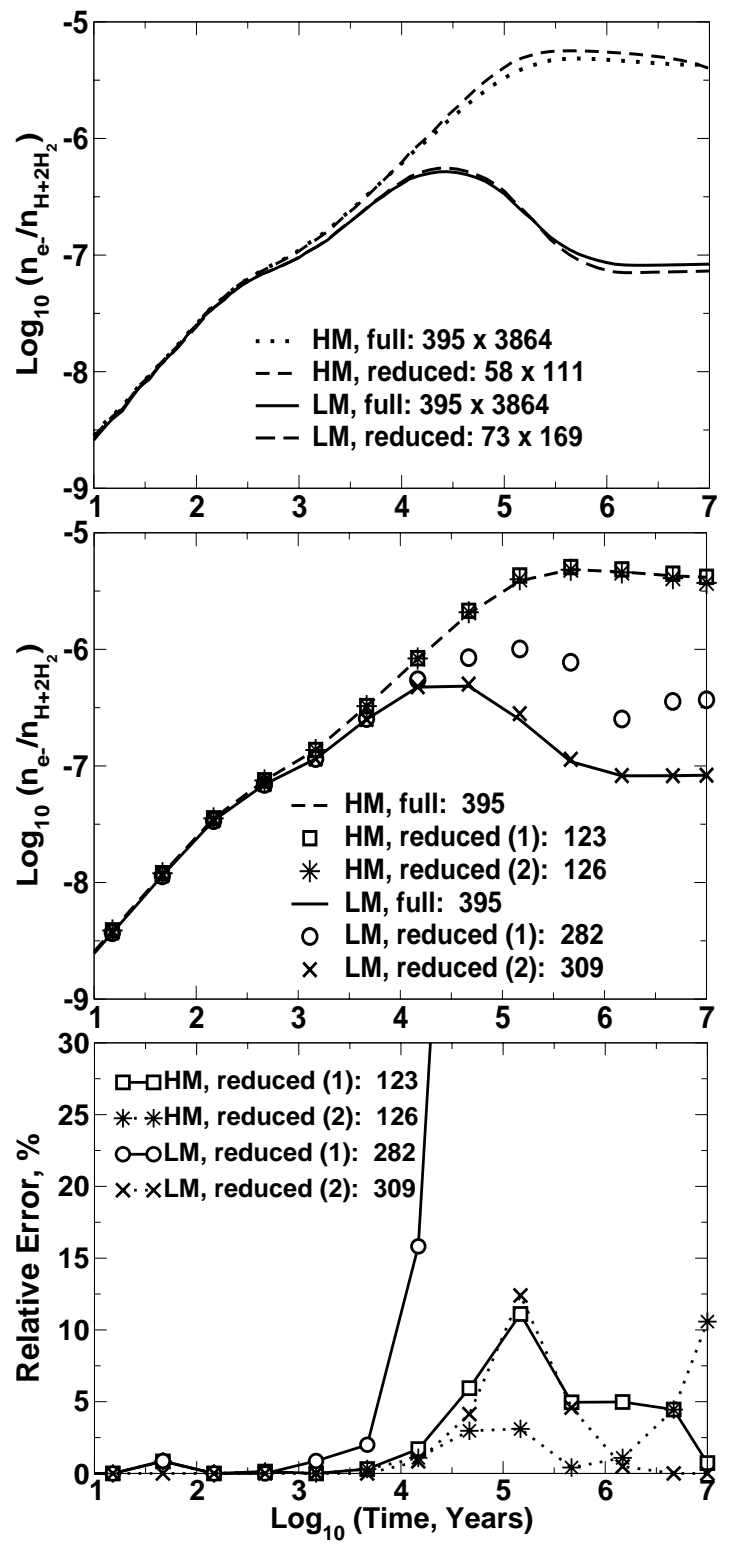

Fig. 3. Ionisation degree (top and middle panels) and relative errors (bottom panel) in a diffuse cloud, computed with the full and reduced networks for pure gas-phase chemistry (HM-GAS-DIFF and LM-GAS-DIFF models). Top panel reproduces results of the reactionbased reduction. Indicated are the "exact" solutions for high (dotted line) and low (solid line) metallicities as well as the solution for the reduced species sets (HM - dashed line, LM - long-dashed line). Middle and bottom panels illustrate the results of the species-based reduction. Shown are the "exact" solutions (HM - dashed line, LM - solid line) and the solutions for various reduced sets (see text).

dominant ions during most of the $10^{7}$ years are $\mathrm{S}^{+}$and $\mathrm{Mg}^{+}$. We found that it is sufficient to consider only these ions as important species in the species-based reduction method. Taking sulphur to be the only important species, we can reduce the number of species from 395 to 123 and achieve a computational speed gain of a factor of about 25 with uncertainty of $\sim 15 \%$ at $t \sim 10^{5}$ years (see Fig. 3, middle and bottom panels, squares). If we consider both ionised magnesium and ionised sulphur to be important species, the number of species in the reduced set 
is 126 , and the accuracy is better than $5 \%$ during the entire evolution (see Fig. 3, middle and bottom panels, stars). The computational gain is almost the same (see Table 3). Note that metals other than magnesium (i.e., sodium and iron) are almost equally important for the ionisation degree. But as they enter the chemical network in a similar way, it is sufficient to designate magnesium to be an important species in order to include in the reduced set all the species that are needed to compute abundances of $\mathrm{Na}$ and $\mathrm{Fe}$ as well.

With the reaction-based reduction technique, we choose electrons to be the only important species. In this case the number of necessary species is 58 , which is more than two times smaller compared to the species-based reduction. Additionally, the number of chemical reactions is reduced from 3864 to 111 (see Fig. 3, top panel). The achieved computational time gain is of the order of 500, while the resulting uncertainties are by definition smaller than $30 \%$ during all $10^{7}$ years of the evolution (see Table 3). It should be recalled that the accuracy is preset in the reaction-based reduction, so in all figures we only show relative errors for the species-based technique.

The small number of necessary reactions in the reduced network can be explained by the fact that in this case the chemistry is governed by a rather restricted set of ionisationrecombination processes. Such a set is easily extracted from the entire network by the reaction-based selection algorithm. The fundamental reactions during the entire evolution time are the ionisation of molecular hydrogen and helium by cosmic rays and ionisation of carbon and sulphur by cosmic ray induced UV photons. These ions rapidly react with abundant molecules by efficient ion-molecule reactions, forming complex molecular ions, like $\mathrm{HCO}^{+}$. In turn, these molecular ions transfer charge to metal atoms. As metal ions are less chemically active, they become the most abundant charged species, regulating the fractional ionisation (Oppenheimer \& Dalgarno 1974).

The situation is more complicated from the chemical point of view when the initial metallicity is low. In the absence of abundant metals, the role of electron suppliers goes to $\mathrm{H}_{3}^{+}$, $\mathrm{HCO}^{+}$, and $\mathrm{C}^{+}$, involved in a rich chemistry. Even though $\mathrm{H}_{3}^{+}$is the dominant ion, it alone does not determine the ionisation degree with enough accuracy which is illustrated in Fig. 3 (middle and bottom panels, circles). When this ion only is considered to be an important species in the species-based reduction, errors are negligible only at earlier times when the chemistry is relatively simple. However, after $10^{4}$ years the errors grow significantly, exceeding a factor of 3 at the end of the computation. To account for later chemistry, one has to take into account another important ion, $\mathrm{HCO}^{+}$. When these two species are designated as important, errors do not exceed $15 \%$ during the entire computational time (crosses in middle and bottom panels in Fig. 3). However, due to the complicated chemical connections of the two ions, the species set reduction is only modest in this case. Less than one hundred species can be excluded from the set with a reduction of the computational time by a factor of 3 only (see Table 3 ).

Not all the reactions involving these ions are important, though. With the reaction-based reduction method, we succeed in isolating a three time smaller set of 73 species involved in 169 reactions which are needed to follow the evolution of the ionisation degree in this case. It decreases the time, needed for the computation, by a factor of 500 (see Table 3). The slightly larger number of the necessary species and reactions in this case compared to the case of high metal initial abundances can be explained by more diverse chemistry involving interactions of complex species. The accuracy of the computed ionisation degree is again better than $30 \%$.

Inclusion of accretion and desorption processes as well as surface reactions into these models does not lead to any noticeable changes, primarily, because the density is too low to produce significant ice mantles. Results of the species-based method show that it is possible to use the same reduced set in HM-GAS-DIFF, HM-GAD-DIFF, and HM-DUST-DIFF models without significant loss of accuracy. The same is true for the low metallicity models. However, applying the reaction-based approach to the gas-grain chemical network including surface reactions (DUST-DIFF), we found only moderate reduction as nearly half of the species have to be retained in the reduced network. The reason is that the surface reactions have very high rates compared to the gas-phase reactions and due to the selectivity of the reaction-based method many of them are designated to remain in the reduced network. This leads to the larger number of chemical species and reactions in such a network compared to the pure gas-phase case and, consequently, to the smaller computational time gains, which are about a factor of ten in this case (see Table 3).

\subsection{Dense cloud}

\subsubsection{Gas-phase chemistry}

When no gas-dust interaction is taken into account, the case of a dense cloud $\left(n_{\mathrm{H}}=10^{7} \mathrm{~cm}^{-3}\right)$ at high metallicity is qualitatively similar to the low-density case. Metals are again the dominant ions. To compute their abundances (and hence the ionisation degree) with better than $50 \%$ mean accuracy one needs to include sodium only to the list of important species in the case of the species-based reduction. The reduced set contains 72 species and provides two orders of magnitude gain in computational speed. To further improve accuracy we add sulphur to the list of important species. With the reduced set of 121 species we obtain errors less than $10 \%$ during the entire computational time. The reaction-based technique gives similar results and produces a reduced network consisting of 131 species and 313 reactions with the same computational gain. The difference between the high- and low-metallicity cases is smaller for a dense cloud than for a diffuse cloud. In a denser environment such chemically active ions as $\mathrm{H}_{3}^{+}$and $\mathrm{HCO}^{+}$are less abundant, and metal ions dominate the ionisation degree both in high- and low-metallicity cases. As a result of this similarity, in a dense cloud for the high and low metallicities the reduced species sets are nearly the same both for the species- and reaction-based approaches, with similar errors and computational gains (see Table 4). 
Table 4. Reductions made for the ionisation degree in a dense cloud with pure gas-phase chemistry.

\begin{tabular}{lllc}
\hline \hline Model & $\begin{array}{l}\text { Important } \\
\text { species }\end{array}$ & $\begin{array}{l}\text { Reduced } \\
\text { network }\end{array}$ & $\begin{array}{c}\text { Speed } \\
\text { gain }\end{array}$ \\
\hline \multicolumn{4}{c}{ Species-based reduction } \\
\hline HM-GAS-DENS & $\mathrm{Na}^{+}$ & $72 \times 568$ & $\sim 100$ \\
HM-GAS-DENS & $\mathrm{Na}^{+}, \mathrm{S}^{+}$ & $121 \times 1560$ & $\sim 30$ \\
LM-GAS-DENS & $\mathrm{Na}^{+}$ & $71 \times 567$ & $\sim 100$ \\
LM-GAS-DENS & $\mathrm{Na}^{+}, \mathrm{S}^{+}$ & $132 \times 1717$ & $\sim 25$ \\
\hline \multicolumn{4}{c}{ Reaction-based reduction } \\
\hline HM-GAS-DENS & $\mathrm{e}^{-}$ & $131 \times 313$ & 130 \\
LM-GAS-DENS & $\mathrm{e}^{-}$ & $157 \times 386$ & 55 \\
\hline
\end{tabular}

\subsection{Gas-grain chemistry}

The situation changes drastically, when gas-grain interactions are taken into account. In the high-metallicity case, at early stages of the evolution, the dominant ions are the same as in the diffuse medium, i.e., metals. However, after $10^{4}$ years their depletion becomes important, and the dominant ions are $\mathrm{H}_{3}^{+}$, $\mathrm{HCO}^{+}$, and $\mathrm{N}_{2} \mathrm{H}^{+}$. The straightforward use of the species-based criterion under such conditions leads to the significant loss of accuracy at this time. The problem is that this criterion is based on the selection of species whose abundances are needed to compute the abundance of an important species. But the depletion case is not that simple. Abundances of mantle species with high desorption energies are definitely not important and do not have to be computed with any accuracy. Based on the formal criterion (10), these species are to be excluded from the reduced set. However, this leads to the exclusion of the corresponding accretion reactions from the network, and counterparts of the excluded mantle species remain in the gas phase, significantly altering its chemistry.

With the species-based reduction method we investigated the GAD-DENS model, i.e., the model in which accretion and desorption processes are taken into account in addition to gasphase chemistry. As we have no formal way to select species whose absence in the gas phase is important to compute the ionisation degree, we choose to check two artificial possibilities. First, we add surface metals to the reduced network as they are primary contributors to the electron abundance in the gas phase. Second, we allow all the selected neutral species to stick to dust grains, reasoning that if their gas phase abundances are deemed important then their depletion is important as well. Results are shown in Fig. 4 (middle and bottom panels). When only metal depletion (with a handful of other species selected automatically) is taken into account the errors are quite large, sometimes reaching $50 \%$. To decrease errors, depletion of all selected neutral species is needed. In the latter case, the approximate solution differs from the "exact" one by as much as $20 \%$ but later on the difference is much smaller dropping to just a few per cent at $t=10^{7}$ years (see Table 5).

The ambiguity of the previous selection procedure is not an issue in the reaction-based approach which is specifically designed to find necessary formation and destruction pathways

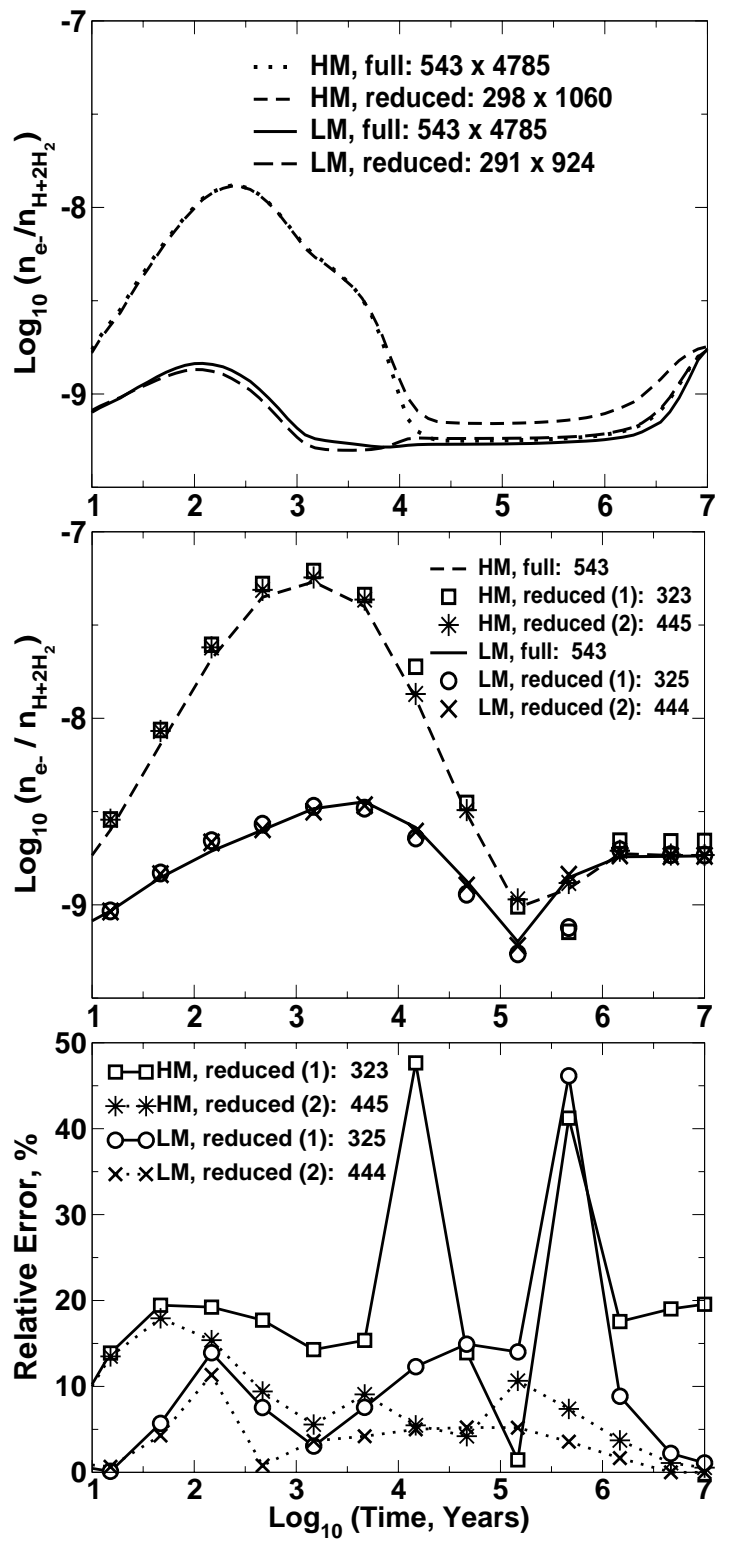

Fig. 4. Ionisation degree (top and middle panels) and relative errors (bottom panel) in a dense cloud, computed with the full and reduced networks for gas-phase chemistry including accretion and desorption processes and dust surface reactions. Top panel reproduces results of the reaction-based reduction for the DUST-DENS model. Indicated are the "exact" solutions for high (dotted line) and low (solid line) metallicities as well as the solution for the reduced species sets (HM dashed line, LM - long-dashed line). Middle and bottom panels illustrate results of the species-based reduction for the GAD-DENS model. Shown are the "exact" solutions (HM - dashed line, LM - solid line) and the solutions for various reduced sets (see text).

rather than necessary species. As it was mentioned above, this method of reduction treats gas-grain interactions and surface reactions equally with gas-phase reactions. Thus, one does not have to worry about which surface species to keep in a reduced set in order to estimate the abundances of important species. Therefore, this method works much better in the reduction of chemical networks involving complicated processes like gas-grains interactions and surface reactions. With the 
Table 5. Reductions made for the ionisation degree in a dense cloud with gas-grain chemistry included.

\begin{tabular}{llll}
\hline \hline Model & $\begin{array}{l}\text { Important } \\
\text { species }\end{array}$ & $\begin{array}{l}\text { Reduced } \\
\text { network }\end{array}$ & $\begin{array}{c}\text { Speed } \\
\text { gain }\end{array}$ \\
\hline \multicolumn{4}{c}{ Species-based reduction } \\
\hline HM-GAD-DENS & $\begin{array}{l}\mathrm{H}_{3}^{+}, \\
\text {surface metals }\end{array}$ & $323 \times 3201$ & $\sim 6$ \\
HM-GAD-DENS & $\begin{array}{l}\mathrm{H}_{3}^{+}, \\
\text {all surface }\end{array}$ & $445 \times 3445$ & $\sim 2$ \\
LM-GAD-DENS & $\begin{array}{l}\mathrm{H}_{3}^{+}, \\
\text {surface metals }\end{array}$ & $325 \times 2933$ & $\sim 4$ \\
LM-GAD-DENS & $\begin{array}{l}\mathrm{H}_{3}^{+}, \\
\text {all surface }\end{array}$ & $444 \times 3171$ & $\sim 2.5$ \\
\hline \multicolumn{3}{c}{ Reaction-based reduction } \\
\hline HM-DUST-DENS & $\mathrm{e}^{-}$ & $298 \times 1060$ & 8 \\
LM-DUST-DENS & $\mathrm{e}^{-}$ & $291 \times 924$ & 7 \\
\hline
\end{tabular}

reaction-based technique, we reduced the number of species and reactions in the gas-grain chemical network involving surface reactions (DUST-DENS). We were able to remove about half of the species from the full gas-grain network and to reduce the number of reactions by a factor of 5 (see Fig. 4, top panel). The reduction speeds up the computation by a factor of 8 only (see Table 5). At times, earlier than about $10^{4}$ years, the ionisation degree is calculated with an accuracy better than $10 \%$. At $t>10^{4}$ years, the uncertainties grow but remain less than $30 \%$. The reason is that it is more difficult to follow the evolution of the ionisation degree with the reduced network, since the importance of the complicated gas-grain interactions and surface reactions is raising up. In the low-metallicity case (LM-GADDENS), which is more complex from the chemical point of view, the reaction-based approach produces a reduced network that consists of 291 species and 924 reactions and predicts an ionisation degree with a requested accuracy of $30 \%$ during the entire evolutionary time. It accelerates the computations by a factor of 7 (see Table 5).

Comparison of top (DUST) and middle (GAD) panels in Fig. 4 shows that there is a difference between the evolution of ionisation degree in the case of gas-grain chemistry with and without surface reactions. When surface reactions are accounted for, fractional ionisation drops to $10^{-9}$ in $t \sim 10^{4}$ years. In the case of gas-phase chemistry with accretion and desorption it reaches the same equilibrium value later, after $10^{5}$ years. Also, the maximum value of the ionisation degree is higher in the latter case. Thus, we conclude that surface reactions are important for the accurate estimation of the fractional ionisation in dense molecular clouds at late stages of their evolution.

To conclude this section, we emphasise that the reactionbased technique is more suitable for the reduction of gas-phase or gas-grain chemical networks if one intends to follow the evolution of the ionisation degree in diffuse or dense molecular clouds with a relatively small number of chemical species and reactions and a reasonable accuracy than it is the case for the species-based scheme.

\section{Carbon monoxide}

Essentially the same scheme of reduction, as in the previous section, is utilised for $\mathrm{CO}$ as well. First, the chemical model is computed with one of the full chemical networks. Its results are used to select key species needed to predict the $\mathrm{CO}$ abundance. Then the validity of a reduced network is checked by comparing the abundances obtained with the full and reduced networks for the entire evolutionary time. In all cases, we assumed that the $\mathrm{CO}$ molecule is the only important species. Results of the reduction and relevant computational speed gains are presented in Tables 6-9.

\subsection{Diffuse cloud}

In the case of pure gas-phase chemistry in a diffuse cloud (at the gas density $n_{\mathrm{H}}=10^{3} \mathrm{~cm}^{-3}$, GAS-DIFF) the main processes, relevant to the evolution of carbon monoxide, are its destruction by cosmic rays or by cosmic ray induced photons and its production through the neutral-neutral reactions of oxygen with carbon atoms and various light carbon-bearing molecules, dissociative recombination of $\mathrm{HCO}^{+}$, and destruction of $\mathrm{H}_{2} \mathrm{CO}$ by $\mathrm{CR}$-induced photons. Since metals as well as $\mathrm{S}, \mathrm{Si}, \mathrm{P}$, and $\mathrm{Cl}$ do not affect the evolution of $\mathrm{CO}$ abundances directly, it is natural to expect that there should not be large differences between the high- and low-metallicity cases.

In the absence of gas-grain interactions the evolution of $\mathrm{CO}$ is comparatively simple - during almost the entire evolutionary time its abundance is steadily approaching the equilibrium value of about $10^{-4}$ which is reached at $\sim 10^{6}$ years (see Fig. 5, top and middle panels).

With the species-based reduction, of all 395 species we select only 36 species in the high-metallicity case and 27 species in the case of low metal abundances (see Fig. 5, middle panel). Due to the small size of these reduced networks, time needed to solve the relevant ODE system is negligible, less than a few seconds (see Table 6). At early evolutionary phase $(t \sim$ $10^{3}-10^{4}$ years) the uncertainties of the $\mathrm{CO}$ abundance can reach $50 \%$ for high metallicity and $80 \%$ for low metallicity. Later, when the equilibrium abundance is reached, error drops to about $20 \%$ (see Fig. 5, bottom panel). The reason for the high uncertainty at early times is that such rare species as $\mathrm{CH}_{3} \mathrm{CO}^{+}$ and $\mathrm{CH}_{2} \mathrm{CO}$, which are not recognised by the species-based algorithm to be necessary species, are in fact important at the early evolutionary phase for the accurate estimation of the $\mathrm{CO}$ abundance.

Applying the reaction-based reduction, we are able to follow the evolution of the $\mathrm{CO}$ abundance for $10^{7}$ years with an accuracy better than $30 \%$. However, the price to pay for this is a significant number of species to be held in the corresponding reduced networks. One needs to keep 158 and 95 species in the reduced networks for high and low metallicities, respectively (see Fig. 5, top panel). The relevant computational speed gain is a factor of 50 for the HM case and about 200 for the LM case (see Table 6). It can be seen from Fig. 5 (top and middle panels), that the $\mathrm{CO}$ abundances in the case of high- and lowmetallicities do behave similarly. Nevertheless, from the chemical point of view the case of low initial abundances of metals 


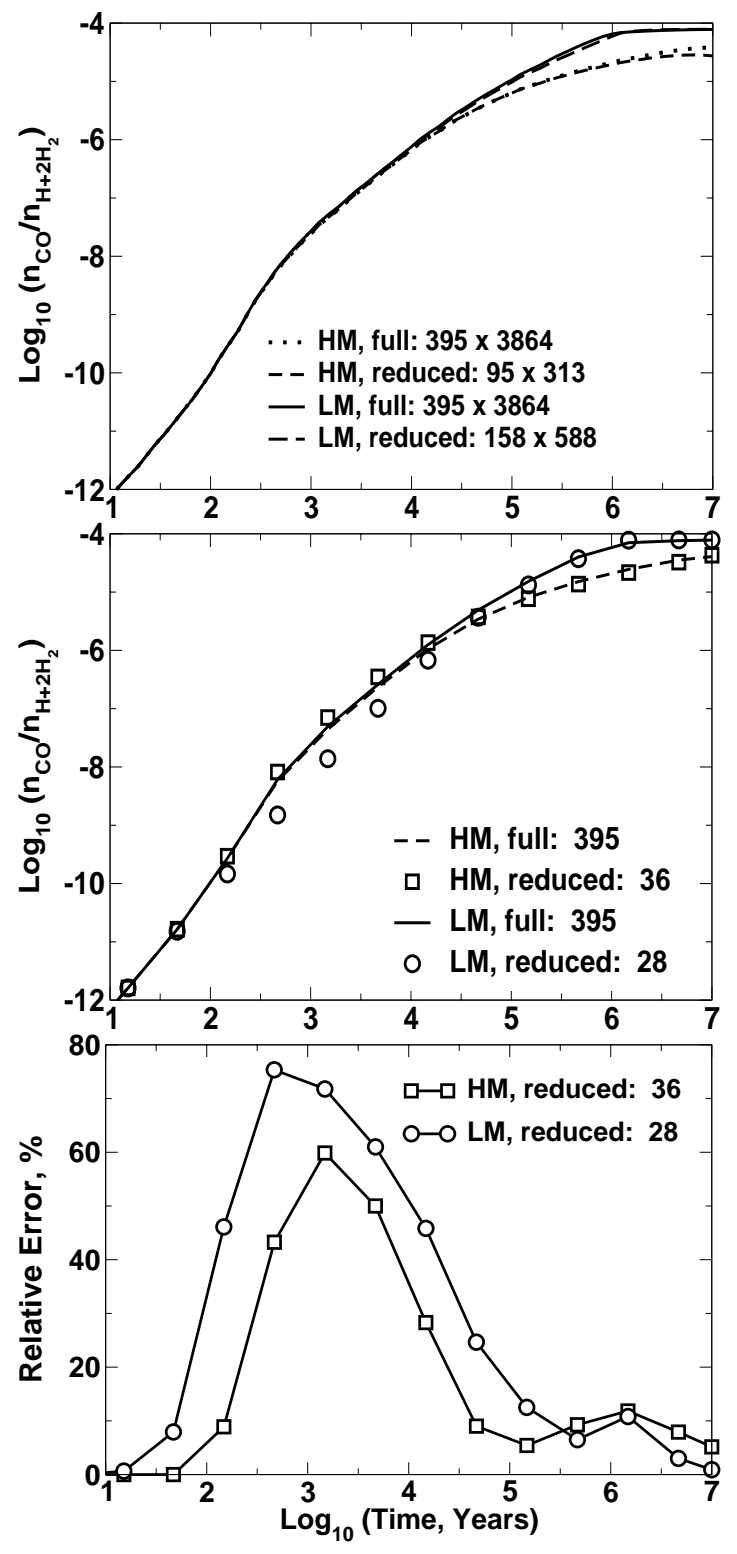

Fig. 5. The CO abundances (top and middle panels) and relative errors (bottom panel) in a diffuse cloud, computed with the full and reduced species set for pure gas-phase chemistry (GAS-DIFF model). $T o p$ panel reproduces results of the reaction-based reduction. Indicated are the "exact" solutions (HM - dotted line, LM - solid line) and the solution for the case when $\mathrm{CO}$ is considered to be the only important species (HM - dashed line, LM - long-dashed line). The middle and bottom panels illustrate results of the species-based reduction. Shown are the "exact" solutions (HM - dashed line, LM - solid line) and the solution for the case when $\mathrm{CO}$ is an important species (HM - open squares, LM - open circles).

is somewhat simpler in comparison with the high metallicity case and implies smaller number of species and reactions to be kept in the reduced network.

Inclusion of accretion-desorption processes and surface reactions does not affect the chemistry in a diffuse cloud significantly. As we already mentioned in Sect. 4.1, the gas-grain interactions are not efficient at such low density $\left(n_{\mathrm{H}}=10^{3} \mathrm{~cm}^{-3}\right)$ since the typical rate of collisions between dust grains and gas
Table 6. Reductions made for $\mathrm{CO}$ in a diffuse cloud.

\begin{tabular}{lccc}
\hline \hline Model & $\begin{array}{l}\text { Important } \\
\text { species }\end{array}$ & $\begin{array}{l}\text { Reduced } \\
\text { network }\end{array}$ & $\begin{array}{c}\text { Speed } \\
\text { gain }\end{array}$ \\
\hline \multicolumn{4}{c}{ Species-based reduction } \\
\hline HM-GAS-DIFF & CO & $36 \times 240$ & $>100$ \\
LM-GAS-DIFF & CO & $28 \times 182$ & $>100$ \\
HM-GAD-DIFF & CO & $37 \times 242$ & $>100$ \\
LM-GAD-DIFF & CO & $27 \times 173$ & $>100$ \\
\hline \multicolumn{4}{c}{ Reaction-based reduction } \\
\hline HM-GAS-DIFF & CO & $158 \times 588$ & 50 \\
LM-GAS-DIFF & CO & $95 \times 313$ & 240 \\
HM-DUST-DIFF & CO & $298 \times 1293$ & 8 \\
LM-DUST-DIFF & CO & $306 \times 1310$ & 5 \\
\hline
\end{tabular}

species is far too low. Using the species-based approach, we found that it is enough to retain only 37 species for the high metal initial abundances and 27 species in the LM case (see Table 6). These are almost the same numbers as in the case of pure gas-phase chemistry. In fact, one can use the same reduced sets of species and reactions in the GAS-DIFF, GADDIFF, and DUST-DIFF models (separately for each metallicity set). However, uncertainties in the computed $\mathrm{CO}$ abundances in all cases exceed $50 \%$ at $t \sim 10^{3}-10^{4}$ years.

It is not that easy to remove these uncertainties. The application of the reaction-based method allows to cut the entire 543 species set by a factor of 2 in this case (see Table 6). It is more than two times the number of species found to be necessary for the correct reproduction of $\mathrm{CO}$ abundances in the case of pure gas-phase chemistry. Consequently, it speeds up the calculations by a factor of 5 only. The reason is, as noted in the previous section, that the reaction-based algorithm tends to keep some surface reactions in the reduced networks. Obviously, it leads to the larger number of species to be retained in the relevant reduced networks compared to the case of pure gas-phase chemistry.

\subsection{Dense cloud}

\subsubsection{Gas-phase chemistry}

In a dense molecular cloud $\left(n_{\mathrm{H}}=10^{7} \mathrm{~cm}^{-3}\right)$ the main processes leading to the production and destruction of $\mathrm{CO}$ molecules in the case of pure gas-phase chemistry (GAS-DENS) are the same as in a diffuse cloud, but with one important change. At such high density, the role of chemical reactions involving abundant species like $\mathrm{C}, \mathrm{O}, \mathrm{CH}$, and $\mathrm{CH}_{2}$, becomes even more important. Therefore, we may expect that in this case the chemistry relevant to the evolution of carbon monoxide is even simpler than in the diffuse cloud (GAS-DIFF). It implies a smaller set of necessary species.

The results of reduction for this case are shown in Fig. 6. The $\mathrm{CO}$ abundance increases rapidly at early evolutionary stages $\left(t \lesssim 10^{3}\right.$ years). Later, when the chemical equilibrium is reached, it remains constant around $10^{-4}$. 


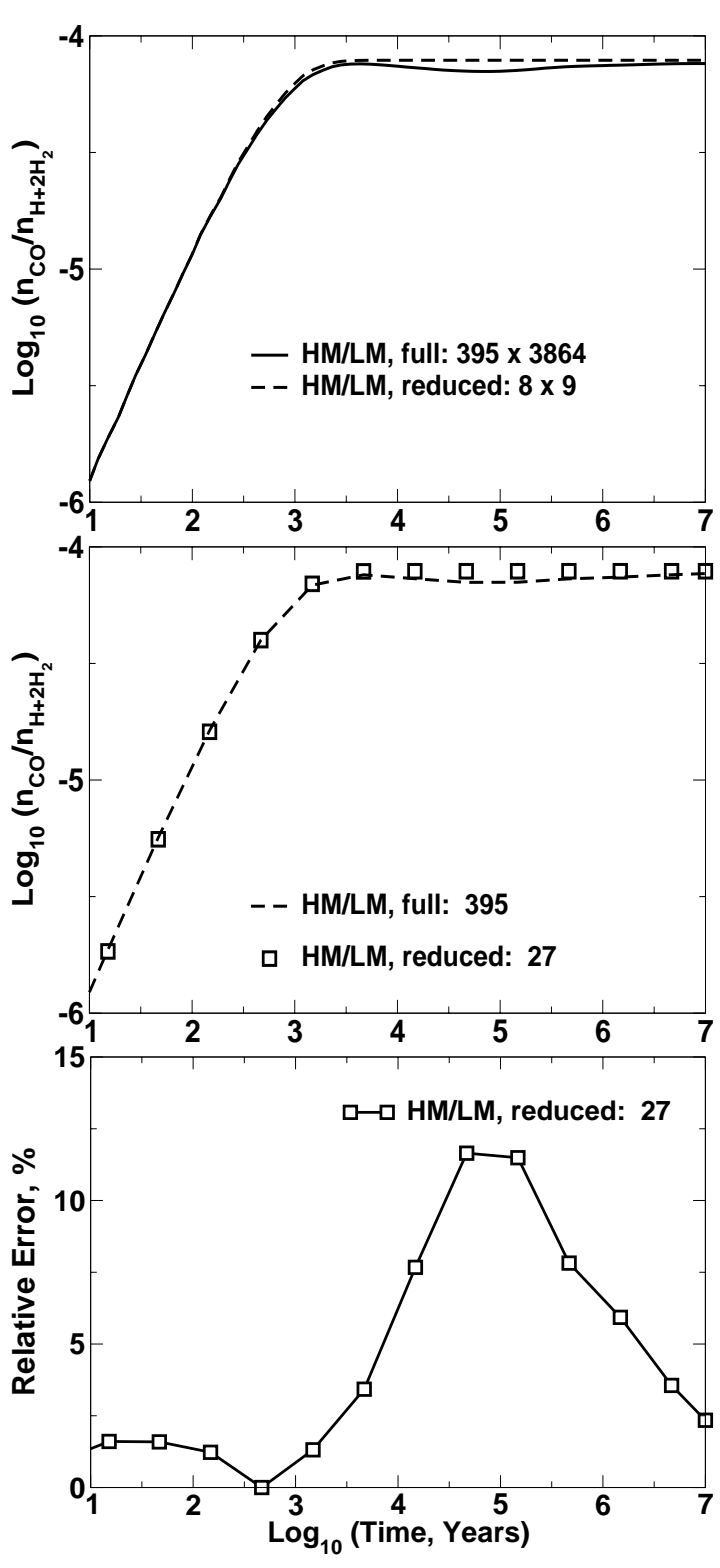

Fig. 6. Same as in Fig. 5 but for a dense cloud (GAS-DENS).

Using the species-based reduction technique, we succeeded in the isolation of a small set of 27 species allowing us to follow the evolution of $\mathrm{CO}$ with a very good accuracy $<15 \%$ for all $10^{7}$ years for both the high- and low-metallicity case (see Fig. 6, middle and bottom panels). Again, the computational time is negligibly small, less than a second (see Table 7).

But even this 27 species set contains excessive information. Applying the reaction-based reduction approach, we revealed that in reality there are only 8 species involved in 9 reactions which are necessary for the accurate estimation of $\mathrm{CO}$ abundances during the entire evolutionary time (see Fig. 6, top panel). The relevant reduced network is given in Table 8 . The uncertainties of computed $\mathrm{CO}$ abundances do not exceed $15 \%$ for all $10^{7}$ years of the evolution. This remarkable result, showing the power of the reaction-based technique, explains why there is no difference between the cases of high and low metals in a dense cloud: metals are completely excluded from this network.

In the medium with the atomic initial composition (except for molecular hydrogen) reactions start from the dissociation of $\mathrm{H}_{2}$ molecules by cosmic ray particles and from their radiative association with atomic carbon. The latter reaction leads to $\mathrm{CH}_{2}$ formation. This molecule reacts with atomic $\mathrm{H}$ and $\mathrm{C}$ leading to $\mathrm{CH}$ and $\mathrm{C}_{2} \mathrm{H}$. These three molecules $\left(\mathrm{CH}, \mathrm{CH}_{2}\right.$, and $\mathrm{C}_{2} \mathrm{H}$ ) associate with atomic oxygen finally producing the $\mathrm{CO}$ molecule. These processes are balanced by destruction of $\mathrm{CO}$ and $\mathrm{CH}$ by $\mathrm{CR}$-induced photons. In this way, the key reaction pathways can be isolated for which high-quality experimental reaction rate measurements have to be provided for an accurate modelling. It may seem to be somewhat surprising that the reduced network does not contain $\mathrm{HCO}^{+}$, at variance with the diffuse cloud case. Note, however, that the maximum value of the $\mathrm{HCO}^{+}$abundance reached in the high-density model $\left(\sim 10^{-10}\right)$ is two orders of magnitude less than in the diffuse cloud model. Given the correspondingly low electron abundance, this prevents effective $\mathrm{CO}$ synthesis in dissociative recombination of $\mathrm{HCO}^{+}$. Proton transfer reactions of $\mathrm{CO}$ with $\mathrm{H}_{2}^{+}$and $\mathrm{H}_{3}^{+}$that would transform $\mathrm{CO}$ to $\mathrm{HCO}^{+}$are also nearly quenched due to low abundances of these ions. Thus, while CO is definitely an important species for $\mathrm{HCO}^{+}$in a dense medium, the opposite is not true.

Going somewhat out of the scope of the present paper, we mention briefly that this network proved to be valid under a much wider range of conditions that are described here. Calculations that will be presented in a subsequent paper show that it can be used for estimating the $\mathrm{CO}$ abundance not only in dark dense clouds, but in a translucent $\left(A_{\mathrm{V}}>1 \mathrm{mag}\right)$ medium as well. It gives less than $50 \%$ error at $10^{4}<n_{\mathrm{H}}<10^{10} \mathrm{~cm}^{-3}$ and $T<250 \mathrm{~K}$ and can be used in a denser medium as well $\left(n_{\mathrm{H}}<10^{12} \mathrm{~cm}^{-3}\right)$ provided $T \sim 200-250 \mathrm{~K}$ and $A_{\mathrm{V}}>5 \mathrm{mag}$, conditions being typical of hot cores or protoplanetary discs.

The accuracy of this network is also insensitive to the variation of the adopted $\mathrm{C} / \mathrm{O}$ ratio $(0.43)$ within a factor of a few. The value of this ratio would be critical, though, in the attempt to construct a reduced network for carbon or oxygen separately. If carbon is less abundant than oxygen (as in the adopted abundance sets), all $\mathrm{C}$ atoms are locked in $\mathrm{CO}$ molecules, and the reduction is rather effective. In fact, it is possible to construct a single network of a few tens of species that reproduces abundances of $\mathrm{C}, \mathrm{O}$, and CO simultaneously with less than $100 \%$ uncertainty. If the $\mathrm{C} / \mathrm{O}$ ratio is greater than 1 , extra carbon is available for producing long carbon chains, and the effective reduction (with $\mathrm{C}$ as an important species) is not possible.

\subsubsection{Gas-grain chemistry}

The situation is no longer trivial when gas-grain interactions and surface reactions are taken into account (GAD-DENS and DUST-DENS). The influence of these processes on the evolution of abundances is twofold. First, the freeze-out of the gasphase $\mathrm{CO}$ leads to the activation of certain chemical reaction chains that are suppressed in the presence of CO. Second, the presence of $\mathrm{CO}$ in icy mantles can drive some surface reactions 
Table 7. Reductions made for $\mathrm{CO}$ in a dense cloud with pure gas-phase chemistry.

\begin{tabular}{llcc}
\hline \hline Model & $\begin{array}{l}\text { Important } \\
\text { species }\end{array}$ & $\begin{array}{l}\text { Reduced } \\
\text { network }\end{array}$ & $\begin{array}{c}\text { Speed } \\
\text { gain }\end{array}$ \\
\hline \multicolumn{4}{c}{ Species-based reduction } \\
\hline HM-GAS-DENS & CO & $27 \times 133$ & $>100$ \\
LM-GAS-DENS & CO & $27 \times 152$ & $>100$ \\
\hline \multicolumn{4}{c}{ Reaction-based reduction } \\
\hline HM-GAS-DENS & CO & $8 \times 9$ & $>10^{4}$ \\
LM-GAS-DENS & CO & $8 \times 9$ & $>10^{4}$ \\
\hline
\end{tabular}

Table 8. The reduced gas-phase network for CO chemistry in a dense cloud obtained with the reaction-based method.

\begin{tabular}{lll}
\hline \hline & Reactions & \\
\hline $1 \mathrm{H}+\mathrm{CH}_{2}$ & $\rightarrow$ & $\mathrm{CH}+\mathrm{H}_{2}$ \\
$2 \mathrm{H}_{2}+\mathrm{C}$ & $\rightarrow$ & $\mathrm{CH}_{2}+$ PHOTON \\
$3 \mathrm{C}+\mathrm{CH}_{2}$ & $\rightarrow$ & $\mathrm{C}_{2} \mathrm{H}+\mathrm{H}$ \\
$4 \mathrm{O}+\mathrm{CH}$ & $\rightarrow$ & $\mathrm{CO}+\mathrm{H}$ \\
$5 \mathrm{O}+\mathrm{CH}_{2}$ & $\rightarrow$ & $\mathrm{CO}+\mathrm{H}+\mathrm{H}$ \\
$6 \mathrm{O}+\mathrm{C}_{2} \mathrm{H}$ & $\rightarrow$ & $\mathrm{CO}+\mathrm{CH}$ \\
$7 \mathrm{H}_{2}+\mathrm{CRP}^{\mathrm{a}}$ & $\rightarrow$ & $\mathrm{H}+\mathrm{H}$ \\
$8 \mathrm{CH}+\mathrm{CRPHOT}^{\mathrm{b}}$ & $\rightarrow$ & $\mathrm{C}+\mathrm{H}$ \\
$9 \mathrm{CO}+\mathrm{CRPHOT}$ & $\rightarrow$ & $\mathrm{C}+\mathrm{O}$ \\
\hline
\end{tabular}

${ }^{\text {a }}$ Cosmic ray particle.

${ }^{\mathrm{b}}$ CRP-induced photon.

that may eventually result in the formation of complex organic species.

In the absence of surface reactions the evolution of carbon monoxide is simple (see Fig. 7, middle panel). First, its abundance grows steadily, at $t \lesssim 5 \times 10^{3}$ years reaching a value of $\sim 5 \times 10^{-5}$. Then the gas-grain interaction becomes important, and the $\mathrm{CO}$ molecules heavily deplete from the gas phase, forming significant mantles. The chemical equilibrium is reached at $10^{5}$ years with the gas-phase $\mathrm{CO}$ abundance of a few times $10^{-8}$.

The species-based reduction technique isolates two sets to be used in the high- and low-metallicity cases of the GADDENS model. But in fact these sets are very similar to each other, and one can use the same set containing about 30 species with high- and low- initial metallicities both in pure gas-phase models and with accretion/desorption taken into account. The resulting uncertainties are smaller than $10 \%$ for both metallicity cases (see Fig. 7, bottom panel). Again, the computational time which is necessary to compute the evolution of $\mathrm{CO}$ is negligibly small.

The evolution of carbon monoxide in the situation, when surface reactions are considered, is shown in Fig. 7, top panel. The gas-phase CO abundance increases at early times and reaches the value of $\sim 10^{-4}$ at $t \sim 10^{3}$ years. Then, the depletion of $\mathrm{CO}$ becomes efficient. At $t \sim 10^{4}-10^{6}$ years the gas-phase $\mathrm{CO}$ abundance is determined by the balance between accretion
Table 9. Reductions made for $\mathrm{CO}$ in a dense cloud with gas-grain chemistry included.

\begin{tabular}{lllc}
\hline \hline Model & $\begin{array}{l}\text { Important } \\
\text { species }\end{array}$ & $\begin{array}{l}\text { Reduced } \\
\text { network }\end{array}$ & $\begin{array}{c}\text { Speed } \\
\text { gain }\end{array}$ \\
\hline \multicolumn{4}{c}{ Species-based reduction } \\
\hline HM-GAD-DENS & CO & $31 \times 148$ & $>100$ \\
LM-GAD-DENS & CO & $29 \times 133$ & $>100$ \\
\hline \multicolumn{4}{c}{ Reaction-based reduction } \\
\hline HM-DUST-DENS & CO & $388 \times 1563$ & 3 \\
LM-DUST-DENS & CO & $270 \times 798$ & 16 \\
\hline
\end{tabular}

and desorption processes. Its value is almost the same as the equilibrium $\mathrm{CO}$ abundance in the GAD-DENS model, i.e., a few times $10^{-8}$. After $10^{6}$ years surface re-processing of $\mathrm{CO}$ takes over. It is converted to other species, mainly, $\mathrm{CO}_{2}$. At this stage the accretion of $\mathrm{CO}$ is no more balanced by its desorption, and after some time the new, much lower equilibrium value $\sim 10^{-11}$ is established. Note that this behaviour is at least partially caused by our simplified treatment of the gas-dust interactions. We adopt the Hasegawa \& Herbst (1993) value for $\mathrm{CO}$ binding energy which is estimated for $\mathrm{a} \mathrm{SiO}_{2}$ surface. In reality, after the $\mathrm{CO}$ mantle is formed, the binding energy of $\mathrm{CO}$ and other molecules may become smaller, so that desorption is still competitive with surface re-processing.

With the reaction-based reduction, we are able to select sets of 388 species and 1563 reactions for the high-metallicity case and 270 species and 798 reactions for the low-metallicity case. The relevant computational gains are only about 3 and 16 , respectively (see Table 9). The error of the predicted $\mathrm{CO}$ abundances is less than $10 \%$ during the entire evolutionary time. The number of species which are needed to estimate the $\mathrm{CO}$ abundance accurately in the high-metallicity case is again $\sim 50 \%$ larger compared to the low-metallicity case. Reduced networks with smaller number of species are not usable in the calculations in this case. The reason is that the relevant ODE systems are so stiff for the DVODE solver that the computational times are much larger compared to the case of the full chemical network.

Concluding this section, we emphasise that if surface reactions are considered in a chemical network, then its reduction for carbon monoxide is of no practical use as the relevant computational speed gains are too small. However, if accretion and desorption of species are taken into account as the only gas-grain processes, then it is possible to find a few tens in half a thousand species which must be kept in a reduced network in order to follow the evolution of $\mathrm{CO}$ without significant ( $\gtrsim 50-100 \%)$ errors.

\section{Discussion}

The purpose of the present paper is to check whether a typical astrochemical database contains redundant information that can be ignored in certain calculations regarding interstellar chemistry. The primary incentive for our work is the long time 

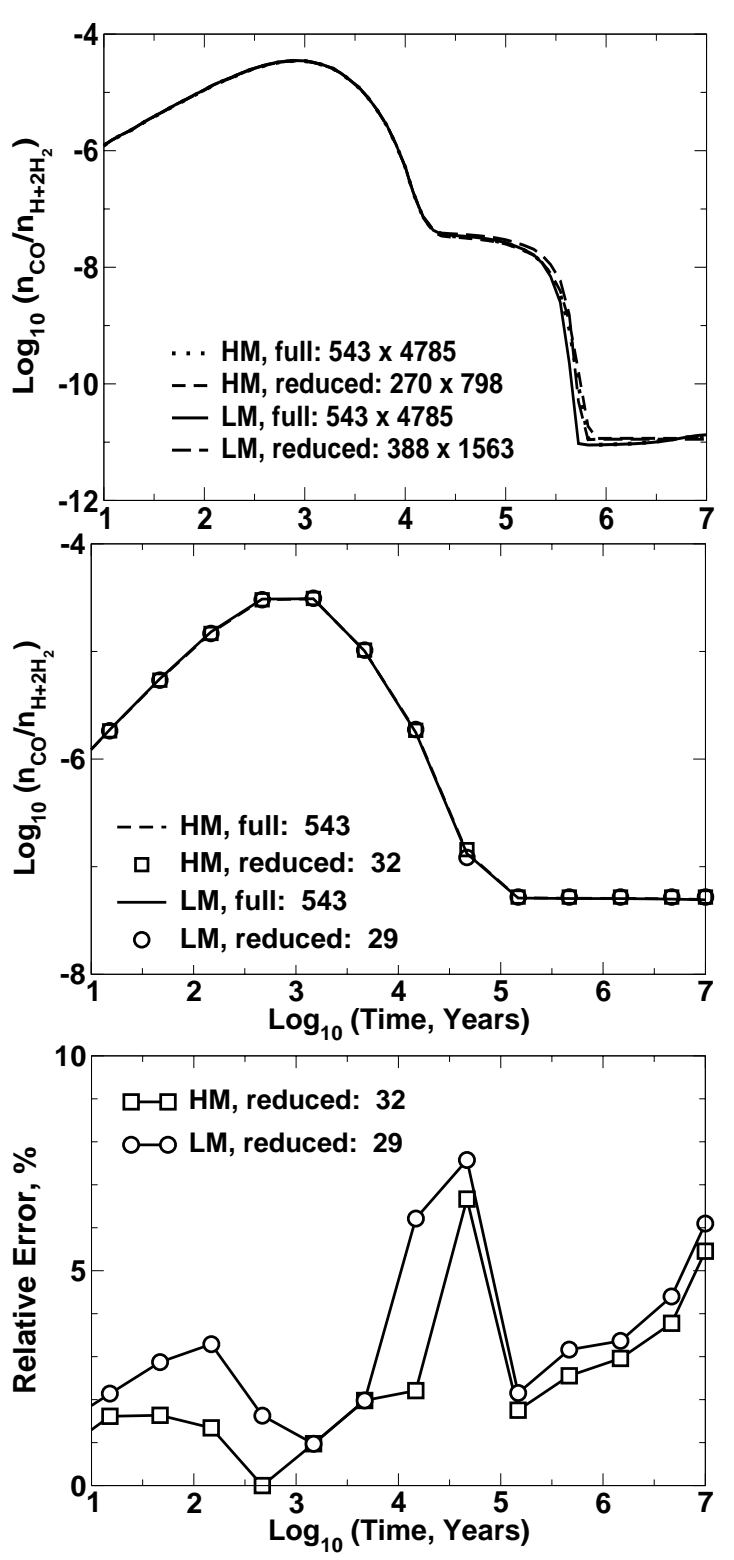

Fig. 7. Same as in Fig. 6 but for the DUST-DENS (top panel) and GAD-DENS (middle and bottom panels) models.

usually needed to complete a more or less detailed model of interstellar chemistry in dynamically evolving media. In many cases the evolution of only a few or even one species are of the main interest for an astronomer. The notable example is the degree of ionisation in dense interstellar clouds which is regulated by the abundances of a few dominant ions. It has been shown by many authors that in this case the relevant chemistry is governed by a rather restricted set of chemical processes (e.g. Oppenheimer \& Dalgarno 1974; Ciolek \& Mouschovias 1995).

Of course, one needs a mathematically rigorous way to "extract" only those species and reactions from the entire network that are needed to reproduce the evolution of a species under investigation within a reasonable numerical accuracy (say, $<100 \%$ ). Such a reduction would decrease the number of species in the network and, hence, the relevant computational time. Using the DVODE solver, we found that this time is proportional to roughly the third power of the number of the involved chemical species. Thus, by decreasing the number of species in the model by just a factor of two, one can obtain an order of magnitude computational speed gain! Besides, the reduction would help to understand the role of a particular chemical compound or reaction for the evolution of an important species. However, one must bear in mind that the cost of the reduction is that the relevant reduced network has a certain range of applicability, i.e. it gives reliable estimates on the abundances of an important species for a specific range of the gas temperature, density, etc.

The first mathematically proven reduction of astrochemical networks is made by RRPHH. Their intention was to isolate a set of species and reactions which are needed to follow the evolution of the $\mathrm{CO}$ abundance within the $30 \%$ accuracy. They considered physical conditions, typical of translucent regions, i.e., $n_{\mathrm{H}}=500-10^{5} \mathrm{~cm}^{-3}, T=10 \mathrm{~K}, A_{\mathrm{V}}=0.5-5 \mathrm{mag}$, with "low metal" initial abundances. They discriminated 69 necessary species participating in 241 reactions from the Ohio New Standard Model, including reactions of dissociative recombination on dust grains. They found that it is possible to decrease further the number of necessary species and reactions to 33 and 116 , respectively, by the artificial modification of the network. It consisted in exclusion of all reactions involving carbon chains longer than $\mathrm{C}_{2} \mathrm{H}_{2}^{+}$and in introduction instead of few synthetic reactions to correctly predict the abundances of shorter carbon chains.

The same method was applied by RBHPR in order to identify reduced networks that predict the degree of ionisation in interstellar clouds with less than $30 \%$ uncertainty under a wide range of physical conditions. They reduced two networks, namely, a "full" network of 218 species and 2747 reactions and a "large" network of 79 species and 1026 reactions. In the "full" network metals beside sodium were excluded, whereas the "large" network in addition excluded nitrogen and most species having more than two nuclei heavier than hydrogen. High and low metal initial abundances were used. They considered the following physical conditions: $T=$ $20 \mathrm{~K}, n_{\mathrm{H}}=500-10^{5} \mathrm{~cm}^{-3}, A_{\mathrm{V}}=1-10 \mathrm{mag}$. The small combined reduced network having 28 species and 71 reactions was found for the case of low metallicity. In the high metallicity case similar network of 29 species and 65 reactions was isolated with the artificially simplified sulphur chemistry.

We analysed the possibility of reducing the number of species and reactions in the UMIST 95 database by two different methods that we refer to as species-based and reactionbased techniques. Our primary goal is to compare the efficiency of these techniques under physical conditions typical of dark regions. We study the applicability of both methods in the case, when the complicated gas-grain interactions and the surface reactions are taken into account. Having in mind possible applications, we tried to make these methods as automatic as possible. Unlike RRPHH and RBHPR, we did not apply artificial modifications to the reduced networks. While such modifications can be useful in certain cases, usually it is not a trivial task and can be fraught with difficulties when a wide range of physical conditions is considered. Thus, we left the reduction entirely to the reduction techniques. 
Both techniques proved to be efficient enough to reduce significantly gas-phase chemical networks needed to compute the ionisation degree or $\mathrm{CO}$ abundance in diffuse and dense molecular clouds with a reasonable accuracy of $<30 \%$. If the accretion-desorption processes and surface reactions are accounted for, the reduction becomes less effective since the relevant chemistry is too complicated. Even though the number of chemical reactions in the reduced networks could be decreased by a few times, making their analysis easier, the computational speed gains are too small in these models to make this reduction of particular interest for chemo-dynamical modelling. Though, at least for the ionisation degree, the situation may be alleviated by the fact that at high densities when depletion is effective, grain charging processes are more important for the fractional ionisation than the chemistry.

We found that the reaction-based technique is more effective compared to the species-based technique (see Tables 3-5). The number of reactions is at least three times smaller for the reaction-based reduction than for the species-based reduction which is natural to expect as reaction rates are not discriminated in the latter method. But the number of species is also smaller for the reaction-based technique in most models. When the gas-dust interaction is taken into account, the reactionbased technique is even more effective than the species-based technique, as it provides the simultaneous reduction of the number of species and reactions and proper treatment of the gas-grain processes. Unfortunately, it is impossible to compare our results for the ionisation degree directly with the results of RBHPR. One reason is that they considered moderate values of visual extinction $A_{\mathrm{V}}=1-10 \mathrm{mag}$, typical of translucent clouds, whereas we dealt with obscured regions, where $A_{\mathrm{V}}>10 \mathrm{mag}$. Another issue is that RBHPR started the reduction from a somewhat smaller "full" network of 218 species and 2747 reactions, while we considered the network of 395 species involved in 3864 reactions. Nonetheless, the resulting reduced networks have comparable sizes, namely, 29 species and 65 reactions (RBHPR, see Table 4) and 58 species and 111 reactions (this work, Table 3 ) in the case of high metals. In the low metallicity case the reduced network by RBHPR has 28 species involved in 71 reactions, compared to our network of 73 species and 169 reactions.

In the case of reduction made for $\mathrm{CO}$, the situation is a bit more diverse. In a diffuse cloud with pure gas-phase chemistry, the species-based reduction technique overcomes the reactionbased method, but at the expense of higher uncertainties (see Table 6 and Fig. 5). In a dense cloud with pure gas-phase chemistry, the reaction-based technique allows us to extract the reduced network which is very small (Table 8 ) but still functional under a very wide range of physical conditions. This network is smaller than the one found by RRPHH, though it has been extracted from the larger full network. Also, it has a wider range of applicability.

The next question to ask is how to apply these methods in dynamical calculations. One can envisage two different strategies to pursue this goal. One way is to reduce the number of species and reactions at the outset and then to use the reduced network during the entire computational time. Another strategy would be to perform the network analysis in the "real-time" mode, during the dynamical evolution.

The first strategy is obviously more cost-effective as it does not add any overhead to the computational time. The reduced network must be prepared in advance and checked against the range of physical conditions that are expected in the dynamical model. Of course, this check is only possible under simplified conditions (otherwise it makes no sense). For example, if the range of densities (temperatures) is expected in the model, one may check that the reduced network is valid at its upper and lower boundaries, hoping that it is valid as well for any densities (temperatures) in between. Of our two techniques, the reaction-based approach works in this mode, as to construct the reduced network the abundances during the entire evolutionary time are analysed. The resultant network accounts for both the early and later chemistry.

The second method seems to be more robust. After the ability of the reduction technique to remove the excessive information from the chemical network is checked, one can perform the reduction, for example, at each dynamical time step or even less often. The full chemical network must be taken into account every time, with current abundances, temperature, density, etc. One may want to identify not only species that can be ignored but species with quasi-stationary abundances as well. Our species-based reduction technique is well suited to be used at a given $t$ with the current values of parameters because, as we mentioned above, the sharp jump in $B_{i}$ values allow to distinguish necessary and unnecessary species clearly. Usually, there is no clear boundary between needed and unneeded reactions (see Fig. 2), so one has to readjust cut-off threshold several times before optimal reduction is found. Thus, in some cases, the reaction-based method is more time-consuming than the species-based approach which is critical if the method is to be invoked from the dynamical code more than once.

Neither method guarantees that the same accuracy, as found in test cases, is preserved during the real computations. With the first method one cannot be sure that conditions, under which the reduced network is not valid, are never met in the dynamical model. The second method properly takes into account the current conditions but is more sensitive to the history of abundance changes. Consider the ionisation degree in the dense cloud when freeze-out is taken into account (high-metallicity case). One of the dominant ions at later times is $\mathrm{N}_{2} \mathrm{H}^{+}$. As a necessary species, it is included in the network at the time when metal depletion becomes important (late stages). However, the abundance of this ion cannot be followed with any accuracy as it is absent in early-time reduced networks, and its entire previous evolution is lost. So, both strategies must be applied with care, taking into account possible uncertainties.

We note in passing that the decreased accuracy of the reduced networks may not be such an issue. Many reactions in the UMIST 95 have poorly measured or estimated rates, with typical uncertainties $\$ 25-100 \%$ or more (Millar et al. 1997). This introduces unknown global errors in the modelled species abundances. On the other hand, modern observations usually provide column densities of abundant species with uncertainties of few tens of percents. Therefore, at nearly all 
astrophysically important situations accuracy of $\sim 50 \%$ seems to be quite appropriate.

\section{Conclusions}

We developed a robust method to reduce simultaneously the number of species and reactions in chemical networks. The straightforward application for such reduced chemical networks would be the modelling of the evolution of magnetised protostellar clouds or protoplanetary discs, when it is necessary to compute the fractional ionisation self-consistently with dynamical processes.

Investigating the applicability of this reduction approach, we considered the conditions of diffuse and dense molecular clouds and utilised the UMIST 95 database of chemical reaction rates. For the sake of comparison, we performed the same reduction with the objective reduction technique. The new reaction-based way of reduction proved to be more efficient and accurate but more time-consuming as well.

It is found that the number of species and reactions that are needed to follow the evolution of the $\mathrm{CO}$ abundance or the ionisation degree can be greatly decreased in the case of pure gas-phase chemistry, implying computational speed gains of more than a few hundred. For instance, to follow accurately (with $<15 \%$ uncertainty) the evolution of the $\mathrm{CO}$ abundance in a dense cloud, it is enough to retain only 8 species involved in 9 reactions in the chemical network. However, as soon as the gas-grain interactions and surface chemistry are taken into account, reduction is modest and corresponding acceleration factors are around 10 .

Acknowledgements. DS was supported by the German Deutsche Forschungsgemeinschaft, DFG project "Research Group Laboratory Astrophysics" (He 1935/17-1), the work of DW was supported by the INTAS grant YS 2001-1/91 and the RFBR grant 01-02-16206. We thank the anonymous referee for comments and suggestions.

\section{Appendix A: Adopted input parameters}

We use the standard DVODE package designed to solve systems of ordinary differential equations. The relevant input parameters adopted in our computations are the following:

- absolute error $\epsilon_{\mathrm{abs}}=2.20 \times 10^{-16}$;

- relative error $\epsilon_{\mathrm{rel}}=10^{-8}$;

- Jacobian computed by the solver (internally).
We found that the relative error $\epsilon_{\text {rel }}=10^{-8}$ is enough to give reliable estimations of the abundances of the species.

We use the $\mathrm{g} 77$ compiler and SuSe Linux 7.3 installed on the Pentium IV $2 \mathrm{GHz}, 1 \mathrm{~GB}$ RAM PC as well as Compaq Visual Fortran 6.5 compiler on Pentium III, $850 \mathrm{MHz}, 256 \mathrm{MB}$ RAM PC. The typical time necessary to run a model is only a few minutes in the case of gas-phase chemistry and can reach half an hour if the gas-grain interactions are taken into account.

\section{References}

Aikawa, Y., \& Herbst, E. 1999, ApJ, 526, 314

Aikawa, Y., Miyama, S. M., Nakano, T., \& Umebayashi, T. 1996, ApJ, 467, 684

Bergin, E. A., \& Langer, W. D. 1997, ApJ, 486, 316

Bergin, E. A., Langer, W. D., \& Goldsmith, P. F. 1995, ApJ, 441, 222

Brown, P. D., \& Charnley, S. B. 1990, MNRAS, 244, 432

Charnley, S. B., Rodgers, S. D., \& Ehrenfreund, P. 2001, A\&A, 378, 1024

Ciolek, G. E., \& Mouschovias, T. Ch. 1995, ApJ, 454, 194

El-Nawawy, M. S., Howe, D. A., \& Millar, T. J. 1997, MNRAS, 292, 481

Gerola, H., \& Glassgold, A. E. 1978, ApJS, 37, 1

Glassgold, A. E., \& Langer, W. D. 1973, ApJ, 179, 147

Hasegawa, T. I., Herbst, E., \& Leung, C. M. 1992, ApJS, 82, 167

Hasegawa, T. I., \& Herbst, E. 1993, MNRAS, 263, 589

Hollenbach, D., \& McKee, C. F. 1979, ApJS, 41, 555

Howe, D. A., Hartquist, T. W., \& Williams, D. A. 1994, MNRAS, 271, 811

Lee, H.-H., Roueff, E., Pineau des Forêts, G., Shalabiea, O. M., et al. 1998, A\&A, 334, 1047

Le Teuff, Y. H., Millar, T. J., \& Markwick, A. J. 2000, A\&AS, 146, 157

Millar, T. J., Rawlings, J. M. C., Bennett, A., Brown, P. D., \& Charnley, S. B. 1991, A\&AS, 87, 585

Millar, T. J., Farquhar, P. R. A., \& Willacy, K. 1997, A\&AS, 121, 139

Oppenheimer, M., \& Dalgarno, A. 1974, ApJ, 192, 29

Rae, J. G. L., Bell, N., Hartquist, T. W., Pilling, M. J., \& Ruffle, D. P. 2002, A\&A, 383, 738 (RBHPR)

Rawlings, J. M. C., Hartquist, T. W., Menten, K. M., \& Williams, D. A. 1992, MNRAS, 255, 471

Ruffle, D. P., Rae, J. G. L., Pilling, M. J., Hartquist, T. W., \& Herbst, E. 2002, A\&A, 381, L13 (RRPHH)

Grevesse, N., \& Sauval, A. J. 1998, Space Sci. Rev., 85, 161

Terzieva, R., \& Herbst, E. 1998, ApJ, 501, 207

Turner, B. E. 2000, ApJ, 542, 837

Umebayashi, T., \& Nakano, T. 1980, PASJ, 32, 405 